\title{
Commentary: Quadriparesis Caused by Lead Poisoning Nine Years after a Gunshot Wound with Retained Bullet Fragments: A Case Report
} Emma Nally ${ }^{1,2}$, Robert D. Bunning ${ }^{1 *}$

'MedStar National Rehabilitation Hospital, 102 Irving St., NW, Washington, DC, 20010, USA ${ }^{2}$ Georgetown University Hospital, Department of Rehabilitation Medicine, Washington, DC, USA

\section{Article Info}

\section{Article Notes}

Received: June 15, 2018

Accepted: July 21, 2018

\section{*Correspondence:}

Dr. Robert D. Bunning, MedStar National Rehabilitation Hospital, 102 Irving St., NW, Washington, DC, 20010, USA

Email: Robert.D.Bunning@Medstar.net

C 2018 Bunning RD. This article is distributed under the terms of the Creative Commons Attribution 4.0 International License.
Despite massive public health efforts and media attention, elevated blood lead levels (BLL) remain a serious public health concern, particularly when they occur in children, who are more susceptible to the toxic effects of the lead. In the 1950's lead was used in water pipes, petroleum, and paint, causing systemic exposure in adults and children, with resultant lead toxicity. Since then, efforts to reduce exposure which has dramatically reduced the incidence of elevated lead levels in the general population. However, there continues to be pandemics and isolated cases of lead poisoning in the United States. In 2015, the water supply in Flint, Michigan was found to be contaminated with lead, and more recently, in 2018, Milwaukee state was found to be mismanaging its childhood lead poisoning prevention program resulting in an increase in the incidence of lead poisonings ${ }^{1,2}$. Although there has been success in lowering environmental levels of lead, research has demonstrated that the level of lead that can cause morbidity is lower than previously believed. In 2012, the reference level for an abnormal concentration of lead was lowered from 10 to $5 \mu \mathrm{g} / \mathrm{dL}^{3}$. This was the latest in a series of changes of successive lowering of the reference levels going back to 1961, when the CDC defined 60 $\mu \mathrm{g} / \mathrm{dL}$ as the reference level ${ }^{4}$. These changes were the result of a better appreciation of the toxicity.

Lead poisoning secondary to retained bullet fragments (RBF) is currently considered a rare phenomenon, even though it is known that lead levels are often elevated in people with $\mathrm{RBF}^{5,6}$. Intraarticular bullet fragments are known to cause elevated lead levels as the $\mathrm{pH}$ in the synovial fluid results in lead leaching from the bullet, and their removal is recommended ${ }^{6}$. However, there has been no consensus on monitoring BLLs in people with extra-articular $\mathrm{RBFs}^{6,7}$. Lead levels have also been found to be elevated in people frequenting shooting ranges due to discharge of lead dust upon firing a bullet with lead primer or containing lead ${ }^{8}$.

The symptoms of lead toxicity are usually vague and non-specific and include abdominal pain, vomiting, constipation, fatigue, and weight loss. Because the occurrence is rare and the symptoms non-specific, the diagnosis of lead toxicity may be delayed, as it was in our case report, or even missed until a post-mortem diagnosis. This delay can result in profound consequences, such as our patient who developed severe motor sensory neuropathy with quadriparesis and allodynia ${ }^{9}$. Long term exposure to lead with BLLs above $5-10 \mu \mathrm{g} / \mathrm{dL}$ has also been 
reported to cause anemia, hypertension, renal dysfunction, and brain injury ${ }^{10}$. It also affects fertility as chronic lead exposure in men reduces fertility and also to increased frequency of miscarriage in women ${ }^{10}$.

At the time of our publication, there was no published protocol for checking lead levels in patients with extrasynovial retained bullet fragments. As in our case and with others, there can be a long delay between the gunshot wound and the onset of lead toxicity. This fact, when coupled with the rarity of the diagnosis, compounds the difficulty in suspecting lead toxicity ${ }^{11,12}$. After our reported case, we measured BLLs in patients admitted to our acute inpatient rehabilitation facility that have non-articular RBF. Thus far we have collected BLLs in five consecutive patients. The time from gunshot wound (GSW) to lead level was an average of 8 years (4 days to 34 years) and we stratified the results by the presence of lead toxicity, co-morbidities, and location of RBF. Lead levels ranged from $2.0 \mu \mathrm{g} / \mathrm{dL}$ to $48.1 \mu \mathrm{g} / \mathrm{dL}$ with $5 / 6$ patients having BLLs above $5 \mu \mathrm{g} / \mathrm{dL}$. The two highest levels (48.0 and $18.7 \mu \mathrm{g} / \mathrm{dL}$ ) were seen in patients with recent GSWs with multiple fragments. 5/6 patients had HTN, 1/6 patients had ESRD, 3/6 patients were anemic, $0 / 6$ patients had documented cognitive dysfunction on a mini-mental status examination ${ }^{13}$. This preliminary data is consistent with the current literature that patients with RBFs are at risk for elevated BLLs and lead toxicity. This supports the need for the development of a protocol for checking BLLs in patients with RBF. Just recently, Nickel et al. recommended that in all cases of extra-articular retained missiles that there be a lead level checked at hospital admission, discharge and monthly intervals until three months' post injury and then again at one year post injury ${ }^{5}$.

GSW injury with RBF is a frequent occurrence in America. In addition, there is an increase in American soldiers with GSWs and injury from improvised explosive devices (IEDs). Retained fragments from either injury may contain lead which may also result in an increase in lead toxicity in this population. The severity of consequences with chronic exposure to lead levels above 5-10 $\mu \mathrm{g} /$ $\mathrm{dL}$, and our preliminary findings as well as the data of frequent elevated BLL in people with $\mathrm{RBF}^{5,6,11,12}$ support the recent recommendation that patients with retained bullet fragments should have periodic assessments of lead levels and be monitored for known clinical symptoms of lead toxicity ${ }^{5}$. Future studies are required to ensure the appropriate time intervals of checking BLLs. Since toxicity can occur from the chronic lead levels as low as $5-10 \mu \mathrm{g} / \mathrm{dL}$, these studies also are needed to determine when treatment, either with chelation therapy or attempted fragment removal, should be undertaken.

\section{Conflict of Interest Statement}

There are no conflicts of interest.

\section{References}

1. Conde X. State Report Finds Milwaukee Mismanaged Its Childhood Lead Poisoning Prevention Program Report Reiterates Findings of Internal Review Published by The Milwaukee Health Department in January. Wisconsin Public Radio. Wisconsin: Board of Regents of the University of Wisconsin System and Wisconsin Educational Communications Board. May 31 2018. Available from: https://www. wprorg/state-report-finds-milwaukee-mismanaged-its-childhoodlead-poisoning-prevention-program

2. Flint Water Crisis Fast Facts. CNN Library. Cable News Network, Turner Broadcasting System, Inc; Updated April 8 2018. Available from: https://www.cnn.com/2016/03/04/us/flint-water-crisis-fastfacts/index.html

3. Lead: Standard Surveillance Definitions and Classifications. CDC Centers for Disease Control and Prevention; updated November $18^{\text {th }}, 2016$. Available from: https://www.cdc.gov/nceh/lead/data/ definitions.htm

4. Gilbert ST, Weiss B. A rationale for lowering the blood lead action level from 10 to $2 \mu \mathrm{g} / \mathrm{dL}$. NeuroToxicology. Sept 2006; Vol 27(5): 693-701.

5. Farrell SE, Vandevander P, Schoffstall JM, et al. Blood lead levels in emergency department patients with retained lead bullets and shrapnel. Acad Emerg Med. March 1999.; Vol 6(3): 208-212.

6. Nickel NW, Steelman TJ, Sabbath ZR, et al. Extra-Articular Retained Missiles; Is Surveillance of the Lead Levels Needed? Military Medicine. March 2018; Vol 183(3-4): e107-e113.

7. DeMartini J, Wilson A, Powell J, et al. Lead Arthropathy and Systemic Lead Poisoning from an Intraarticular Bullet. American Journal of Roentgenology. May 2001; Vol 176(5): 1144-1144

8. Laidlaw M, Filippelli G, Mielke $\mathrm{H}$, et al. Lead exposure at firing ranges - a review. Environmental Health. April 2017; Vol 16(34).

9. Nally E, Jelinek J, Bunning R. Quadriparesis caused by lead poisoning nine years after a gunshot wound with retained bullet fragments: a case report. PM\&R. April 2017; Vol 9(4): 411-414

10. Wani A, Ara A, Usmani A. Lead toxicity: a review. Toxicology. March 2016; Vol 8(2): 55-64

11. Nguyen A, Schaider J, Manzanares M, et al. Elevation of blood lead levels in emergency department patients with extra-articular retained missiles. J Trauma. Feb 2005; Vol58( 2): 289-299

12. McQuirter J, Rothenberg S, Dinkins G, et al. Change in blood lead concentration up to 1 year after a gunshot wound with a retained bullet. Am J Epidemiol. April 2004; Vol 159(7): 683-962

13. Hankenson J, Nally E, Bunning R. Screening for lead toxicity after gunshot wound in a rehabilitation setting. American Academy of Physical Medicine and Rehabilitation Annual Meeting Poster Session. Accepted to present October $25^{\text {th }} 2018$; Orlando Fl. 